\title{
THE ERROR TERM IN NEVANLINNA THEORY. II
}

\author{
SERGE LANG
}

Nevanlinna theory [Ne] was created to give a quantitative measure of the value distribution for meromorphic functions, for instance to measure the extent to which they approximate a finite number of points. We view a meromorphic function as a holomorphic map $f: \mathbf{C} \rightarrow \mathbf{P}^{1}$ into the projective line. The theory has various higher dimensional analogues, of which we shall later consider maps $f: \mathbf{C}^{n} \rightarrow X$ where $X$ is a projective complex manifold of dimension $n$.

We first deal with the classical case of Nevanlinna with $n=1$. Let $a \in \mathbf{P}^{1}$. By a Weil function associated with $a$ we mean a continuous function

$$
\lambda_{a}: \mathbf{P}^{1}-\{a\} \rightarrow \mathbf{R}
$$

having the property that in some open neighborhood of $a$ there exists a continuous function $\alpha$ such that if $z$ is a local coordinate at $a$, then

$$
\lambda_{a}(z)=-\log |z-a|+\alpha(z) .
$$

The difference between two Weil functions is a continuous (and therefore bounded) function on $\mathbf{P}^{1}$. A Weil function roughly measures the distance from $a$. As usual, for real $x>0$ define $\log ^{+}(x)=\max (\log x, 0)$. Let $z$ be the standard coordinate on $\mathbf{C}$. Nevanlinna takes the functions

$$
\begin{gathered}
\lambda_{a}(z)=\log ^{+} 1 /|z-a| \quad \text { if } a \neq \infty, \\
\lambda_{a}(z)=\log ^{+}|z| \quad \text { if } a=\infty .
\end{gathered}
$$

One defines the corresponding mean proximity function

$$
m_{f}\left(\lambda_{a}, r\right)=\int_{0}^{2 \pi} \lambda_{a}\left(f\left(r e^{i \theta}\right)\right) \frac{d \theta}{2 \pi} .
$$

One usually writes $m_{f}(a, r)$ instead of $m_{f}\left(\lambda_{a}, r\right)$ since a definite

Received by the editors April 25, 1989 and, in revised form, July 11, 1989.

1980 Mathematics Subject Classification (1985 Revision). Primary 11J68, 30D35, 32H30. 
Weil function has been chosen once for all. As a function of $r$, $m_{f}(a, r)$ is defined up to $O(1)$, independently of the choice of Weil function.

For $R>1$ we define the normalized zero counting function by

$$
N_{f}(0, R)=\sum_{\substack{a \in \mathbf{D}(R) \\ a \neq 0, f(a)=0}}\left(\operatorname{ord}_{a} f\right) \log \left|\frac{R}{a}\right|+\left(\operatorname{ord}_{0} f\right) \log R,
$$

where $\mathbf{D}(R)$ is the disc of radius $R$, and ord denotes the order of the zero of $f$ at $a$. We define $N_{f}(\infty, R)=N_{1 / f}(0, R)$ and $N_{f}(a, R)=N_{f-a}(0, R)$. Thus $N_{f}(0, R)$ measures the number of zeros of $f$ in the disc of radius $R$, suitably weighted.

One defines the height function associated to $f$ by

$$
T_{f, a}(r)=m_{f}(a, r)+N_{f}(a, r) .
$$

Using Jensen's formula, it is easy to prove that $T_{f, a}$ is independent of a modulo $O(1)$. We write $T_{f}$ instead of $T_{f, \infty}$. We choose $\lambda_{a}$ to make $T_{f, a}$ an increasing function of $r$, and to have certain smoothness properties, by letting for instance

$$
\lambda_{a}(z)=-\frac{1}{2} \log \frac{|z-a|^{2}}{\left(1+|z|^{2}\right)\left(1+|a|^{2}\right)} \text { for } a, z \neq \infty .
$$
Let

Write $f=f_{1} / f_{0}$ where $f_{1}, f_{0}$ are entire without common zero.

$$
W\left(f_{0}, f_{1}\right)=f_{0} f_{1}^{\prime}-f_{0}^{\prime} f_{1}
$$

be the Wronskian. We define the ramification counting function

$$
N_{f, \operatorname{Ram}}(r)=N_{W}(0, r) \text {. }
$$

Basic conditions. Let $a_{1}, \ldots, a_{q}$ be distinct points of $\mathbf{P}^{1}$. Suppose for simplicity that $f(0) \neq 0, \infty$, $a_{j}$ for all $j$, and $f^{\prime}(0) \neq 0$.

Under the basic conditions, Nevanlinna's classical theorem [Ne] is that asymptotically for $r \rightarrow \infty$, we have

$$
(q-2) T_{f}(r)-\sum N_{f}\left(a_{j}, r\right)+N_{f, \operatorname{Ram}}(r)=O\left(\log r+\log T_{f}(r)\right)
$$

except for $r$ lying in a set of finite Lebesgue measure. Nevanlinna also gives explicit constants in the error term on the right.

Osgood [Os 1, Os 2] noticed a similarity between the 2 occurring in Nevanlinna's theorem above on the left-hand side, and the 2 occurring in Roth's theorem [Ro]. However, Vojta [Vo 1] gave a much deeper analysis by pointing out that the whole theory of 
heights in algebraic number theory and diophantine geometry is analogous to Nevanlinna theory. Following this analogy, I looked into the error term of Nevanlinna's theorem [La 5], as follows.

Let $\alpha$ be a real, irrational number. In [La 2] and [La 3] I defined a type for $\alpha$ to be a positive increasing function $\psi$ such that

$$
-\log \left|\alpha-\frac{p}{q}\right|-2 \log q \leq \log \psi(q)
$$

for all but a finite number of fractions $p / q$ in lowest form, $q>0$. The height $h(p / q)$ is defined to be $\log \max (|p|,|q|)$. If $p / q$ is close to $\alpha$, then $\log q$ has the same order of magnitude as the height, so $\log q$ is essentially the height in the above inequality. A theorem of Khintchine states that almost all numbers have type $\psi$ if

$$
\sum_{q=1}^{\infty} \frac{1}{q \psi(q)}<\infty
$$

A basic question is whether Khintchine's principle applies to algebraic numbers, although possibly some additional restrictions on the function $\psi$ might be needed. Roth's theorem can be formulated as saying that an algebraic number has type $\leq q^{\varepsilon}$ for every $\varepsilon>0$, and in the sixties I conjectured in line with Khintchine's principle that this could be improved to having type $\leq(\log q)^{1+\varepsilon}$. Cf. [La 1, La 3, 4] especially. ${ }^{1}$ Thus for instance, we would have the improvement of Roth's inequality

$$
\left|\alpha-\frac{p}{q}\right| \geq \frac{C(\alpha, q)}{q^{2}(\log q)^{1+\varepsilon}}
$$

which could be written

$$
-\log \left|\alpha-\frac{p}{q}\right| 2 \log q \leq(1+\varepsilon) \log \log q
$$

for all but a finite number of fractions $p / q$. However, except for quadratic numbers, which all have bounded type (trivial exercise), there is no example of an algebraic number about which one knows that it is or is not of type $(\log q)^{k}$ for some number $k>1$. It becomes a problem to determine the type for each algebraic number and for the classical numbers. For instance, it follows from Adams' work [Ad 1], [Ad 2] that $e$ has type

$$
\psi(q)=\frac{C \log q}{\log \log q}
$$

\footnotetext{
${ }^{1}$ Unknown to me until much later, similar conjectures were made by Bryuno [Br] and Richtmayer, Devaney and Metropolis [RDM], see [L-T 1] and [L-T 2].
} 
with a suitable constant $C$, which is much better than the "probability" type, and goes beyond Khintchine's principle: the sum $\sum 1 / q \psi(q)$ is divergent.

In light of Vojta's analysis, it occurred to me to transpose my conjecture about the error term in Roth's theorem to the context of Nevanlinna theory.

It becomes a problem to determine the "type" of the classical meromorphic functions, i.e. the best possible error term in the inequality which describes the value distribution of the function. It is classical, and easy, for example, that $e^{z}$ has bounded type, i.e. that the error term in Nevanlinna's theorem is $O(1)$. But I do not even know an example of a function which does not have bounded type! There are two problems here:

- To determine for "almost all" functions (in a suitable sense) whether the type follows the pattern of Khintchine's convergence principle.

- To determine the specific type for each concrete classical function, using the specific special properties of each such function $\wp, \theta, \Gamma, \zeta, J$, etc.

In [La 5] I conjectured a best possible error term, but was not able to prove it exactly. For instance, instead of $1+\varepsilon$ I got only $3 / 2+\varepsilon$. Using a method from Ahlfors' paper [Ah], P. M. Wong [Wo] obtained the error term with $1+\varepsilon$. I pointed out to him that his method would also prove the desired result with an arbitrary type function $\psi$ satisfying only the Khintchine convergence principle. Thus the result precisely stated is as follows.

Let $\psi$ be a positive (weakly) increasing function such that

$$
\int_{e}^{\infty} \frac{1}{u \psi(u)} d u=b_{0}(\psi)
$$

is finite. For any positive increasing function $F$ of class $C^{1}$ such that $r \mapsto r F^{\prime}(r)$ is positive increasing, and for $r, c>0$ we define the error function

$$
S(f, c, \psi, r)=\log F(r)+\log \psi(F(r))+\log \psi(c r F(r) \psi(F(r))) .
$$

We let $r_{1}(F)$ be the smallest number $\geq 1$ such that $F\left(r_{1}\right) \geq 1$, and we let $b_{1}(F)$ be the smallest number $\geq 1$ such that

$$
b_{1} r F^{\prime}(r) \geq e \text { for } r \geq 1 \text {. }
$$

Theorem 1. Under the basic conditions, there are constants $b=$ $b\left(f, a_{1}, \ldots, a_{q}\right)$ and $B_{q}$ (depending on $q$ ) such that for all $r \geq$ $r_{1}\left(T_{f}\right)$ outside a set of measure $\leq 2 b_{0}(\psi)$ and all $b_{1} \geq b_{1}\left(T_{f}\right)$ we 
have

$(q-2) T_{f}(r)-\sum N_{f}\left(a_{j}, r\right)+N_{f, \operatorname{Ram}}(r) \leq \frac{1}{2} S\left(B_{q} T_{f}^{2}, b_{1}, \psi, r\right)+b$.

We can take $B_{q}=12 q^{2}+q^{3} \log 4$, and $b$ can also be determined explicitly.

The $T_{f}^{2}$ already occurs in Ahlfors, but in a form with unspecified constants. Since the dominant term in the error term $S$ is essentially a $\log$, the error term amounts to $\frac{1}{2} \log T_{f}^{2}=\log T_{f}$ in first order approximation. Wong obtained the correct factor $\frac{1}{2}$ by Ahlfors' method, rather than through the singular volume form used previously by other authors.

I shall now describe the result in the higher, equidimensional case, first investigated by Carlson-Griffiths [C-G]. Let

$$
f: \mathbf{C}^{n} \rightarrow X
$$

be a holomorphic map into a compact complex manifold of dimension $n$. We assume that $f$ is nondegenerate, in the sense that the derivative of $f$ at some point is nonsingular. Let $z=$ $\left(z_{1}, \ldots, z_{n}\right)$ be the complex coordinates on $\mathbf{C}^{n}$, and let $\|z\|$ be the euclidean norm. We define the differential forms on $\mathbf{C}^{n}$ :

$$
\omega(z)=d d^{c} \log \|z\|^{2} \text { and } \sigma(z)=d^{c} \log \|z\|^{2} \wedge \omega^{n-1} .
$$

Here $d^{c}=(\partial-\bar{\partial}) / 4 \pi i$ and $d$ is the usual exterior derivative. Note that $\sigma(z)$ in dimension $n=1$ is $d \theta / d \pi$.

Let $D$ be a divisor on $X$, so $D$ is locally a hypersurface. We suppose that $D$ is effective, so $D$ can be represented by one holomorphic function $\varphi$ locally, up to an invertible holomorphic function, and $D$ is defined by $\varphi=0$. By a Weil function associated with $D$ we mean a function

$$
\lambda_{D}: X-\text { support of } D \rightarrow \mathbf{R}
$$

such that if $D$ is represented by $\varphi$ on an open set $U$, there exists a continuous function $\alpha$ on $U$ such that

$$
\lambda_{D}(P)=-\log |\varphi(P)|+\alpha(P) \quad \text { for } P \in U-D .
$$

We define the mean proximity function

$$
m_{f, D}(r)=\int_{\mathbf{S}(r)}\left(\lambda_{D} \circ f\right) \sigma,
$$

where $\mathbf{S}(r)$ is the sphere of radius $r$ in $\mathbf{C}^{n}$. Thus the mean proximity function measures the average approximation of $D$ by the values of $f$ on the spheres. In order to get smoothness, one 
must select smooth Weil functions $\lambda_{D}$ in their class $\bmod O(1)$. This is done as follows.

We let $L_{D}$ be a holomorphic line bundle over $X$ having a meromorphic section $s$ whose divisor $(s)$ is precisely $D$. Since $X$ is compact, such a section is well defined up to a constant factor. We let $\rho$ be a hermitian metric on $L_{D}$. Then we select

$$
\lambda_{D}=-\log |s|_{\rho} .
$$

We define the counting function

$$
N_{f, D}(r)=\int_{0}^{r} \frac{d t}{t} \int_{\left(f^{*} D\right) \cap \mathbf{B}(r)} \omega^{n-1},
$$

where $\mathbf{B}(r)$ is the ball of radius $r$, and we define the height function

$$
T_{f, D}=m_{f, D}+N_{f, D}
$$

Strictly speaking, we should write $T_{f, \rho}$, to indicate that $T$ depends on the metric $\rho$; but a change of $\rho$ changes the height function only by a bounded function. With these differential geometric definitions, $T_{f, D}$ is an increasing function. In fact, if we define the first Chern form outside the support of $(s)$ by the formula

then

$$
c_{1}(\rho)=-d d^{c} \log |s|_{\rho}^{2}
$$

$$
T_{f, \rho}(r)=\int_{0}^{r} \frac{d t}{t} \int_{\mathbf{B}(r)} f^{*} c_{1}(\rho) \wedge \omega^{n-1}
$$
Then

Let $\Omega$ be a volume form on $X$ (i.e. a positive $(n, n)$-form).

$$
f^{*} \Omega=|\Delta|^{2} h \Phi,
$$

where $\Phi$ is the Euclidean volume form on $\mathbf{C}^{n}, h$ is $C^{\infty}$ real $>0$, and $\Delta$ is a holomorphic function on $\mathbf{C}^{n}$, which defines the ramification divisor of $f$. Let $Z$ denote the ramification divisor. We define

$$
N_{f, \operatorname{Ram}}(r)=\int_{0}^{r} \frac{d t}{t} \int_{Z \cap \mathbf{D}(r)} \omega^{n-1} .
$$

We say that a divisor $D$ has simple normal crossings if $D=\sum D_{j}$ is a formal sum of nonsingular irreducible divisors, and locally at each point of $X$ there exist complex coordinates $z_{1}, \ldots, z_{n}$ such that in a neighborhood of this point, $D$ is defined by

$$
z_{1} \cdots z_{k}=0 \text { with } k \leq n .
$$

When $n=1$, then the property of $D$ having simple normal crossings is equivalent to the property that $D$ consists of distinct points, 
taken with multiplicity 1 . The maximal value of $k$ which can occur will be called the complexity of $D$.

Finally, in higher dimension $n$, we suppose that $r \mapsto F(r)$ and $r \mapsto r^{2 n-1} F^{\prime}(r)$ are positive increasing functions of $r$, and we define the error function

$S(F, c, \psi, r)=\log F(r)+\log \psi(F(r))+\log \psi\left(c r^{2 n-1} F(r) \psi(F(r))\right)$.

We let $b_{1}(F)$ be the smallest number $\geq 1$ such that

$$
b_{1} r^{2 n-1} F^{\prime}(r) \geq e \text { for all } r \geq 1 \text {. }
$$

The definition of $r_{1}(F)$ is the same as for $n=1$. Then the analogue of Theorem 1 in higher dimension runs as follows.

Theorem 2. Suppose that $f(0) \notin D$ and $0 \notin \operatorname{Ram}_{f}$. Let $D$ have simple normal crossings, of complexity $k$. Let $K$ be a canonical divisor on $X$. Let $T_{f}=T_{f, E}$ where $E$ is a hyperplane section in some projective imbedding of $X$. Then

$$
\begin{aligned}
T_{f, K}(r)+T_{f, D}(r)-N_{f, D}(r)+N_{f, \text { Ram }}(r) & \\
& \leq \frac{n}{2} S\left(B T_{f}^{1+k / n}, b_{1}, \psi, r\right)+B^{\prime}
\end{aligned}
$$

for all $r \geq r_{1}\left(T_{f}\right)$ outside a set of measure $\leq 2 b_{0}(\psi)$, and some constants $B=B(D, E)$ and $B^{\prime}=B^{\prime}(D, E)$ which can be given explicitly.

The general shape of the theorem stems from Carlson-Griffiths [CG]. I raised the question of a best possible error term in [La 5] but I was not able to prove the conjectured result at that time. By using Ahlfors' method, Wong [Wo] obtained not only the $T_{f}^{2}$ as in Ahlfors, but also the "correct" factor $n / 2$. The final improvement with $1+k / n$ instead of 2 follows from a technical change in Wong's proof at the appropriate moment, and will be given in detail in a forthcoming Springer Lecture Note. I also improved Wong's formulation by using the arbitrary Khintchine type function $\psi$ in the final estimate and by not making any restriction on the divisor other than simple normal crossings. Wong, following some previous authors, assumes unnecessarily that the irreducible components all lie in the same linear system. Otherwise, the general pattern of the proof is due to Wong. It makes use of some ideas of Carlson-Griffiths concerning curvature, but somewhat more efficiently, in a way which should have significance elsewhere in complex differential geometry.

I would conjecture that the exponent $1+k / n$ is best possible. Thus the error term should be determined by local considerations 
on the divisor, in terms of the complexity of its singularities. The conjecture can then be transposed to a strengthening of Schmidt's theorem [Sch]. The exponent $1+k / n$ also applies when there is no divisor $D$, so $k=0$, in which case $T_{f}$ occurs with exponent 1 on the right-hand side. Thus the exponent $1+k / n$ interpolates very neatly between the two extreme cases $D=0$ and $D$ defined locally by $z_{1} \cdots z_{n}=0$.

The same error term can be given in the theorem on the logarithmic derivative, originally stated with a weak error term by Nevanlinna in dimension 1 , and proved using a differential geometric method, $[\mathrm{Ne}]$, p. 259. A higher dimensional version was formulated and proved by Griffiths [Gr] , p. 70, still with a weak error term. The version I now have runs as follows.

Theorem 3. Let $f: \mathbf{C}^{n} \rightarrow X$ be holomorphic nondegenerate. Let $\Psi$ be a meromorphic $n$-form with no zeros on $X$, and such that its polar divisor $D$ has simple normal crossings. Let

$$
f^{*} \Psi=L_{f}(z) d z_{1} \wedge \cdots \wedge d z_{n} .
$$

Define

$$
\nu_{f}(r)=\int_{\mathbf{S}(r)} \log ^{+}\left|L_{f}\right| \sigma .
$$

Let $K$ be a canonical divisor and assume $-K$ is ample. Then for some constants $B, B^{\prime}$ we have

$$
\nu_{f}(r) \leq \frac{n}{2} S\left(B T_{f}^{1+k / n}, b_{1}, \psi, r\right)+B^{\prime}
$$

for all $r \geq r_{1}$ outside a set of measure $\leq 2 b_{0}(\psi)$.

Note that in dimension 1 , taking $X=\mathbf{P}^{1}$ and $\Psi=d z / z$, then $D=(0)+(\infty)$, and $L_{f}$ is the logarithmic derivative

$$
L_{f}=f^{\prime} / f,
$$

so Nevanlinna's classical set up is a special case. The proof follows the pattern of Nevanlinna-Griffiths, but using the Ahlfors technique as revived by Wong.

Finally, the same type of error term can be obtained for holomorphic maps

$$
f: \mathbf{C} \rightarrow X \text { or } f: \mathbf{D}(R) \rightarrow X .
$$

I shall formulate here one version stemming from Griffiths-King [G-K] and Vojta [Vo 1] Theorem 5.7.2. 
Let $Y$ be a complex manifold, and let $f: \mathbf{D}(R) \rightarrow Y$ be a nonconstant holomorphic map. If $\omega$ is a $(1,1)$-form on $Y$ then we define the height

$$
T_{f, \omega}(r)=\int_{0}^{r} \frac{d t}{t} \int_{\mathbf{D}(t)} f^{*} \omega .
$$

We write as usual

$$
f^{*} \omega=\gamma_{f} \Phi, \quad \text { where } \Phi=\frac{\sqrt{-1}}{2 \pi} d z \wedge d \bar{z} .
$$

Then

$$
\gamma_{f}=|\Delta|^{2} h
$$

where $h$ is $C^{\infty}$. If $\omega$ is positive, then $h>0$. The function $\Delta$ is holomorphic and defines the ramification divisor $\operatorname{Ram}_{f}$, which in this case is a discrete set of zeros with multiplicities. We define $\operatorname{Ric} f^{*} \omega=d d^{c} \log h$.

Theorem 4. Let $Y$ be a complex manifold (not necessarily compact). Let $\omega$ be a positive $(1,1)$-form on $Y$ and let $f$ : $\mathbf{D}(R) \rightarrow Y$ be a holomorphic map. Suppose there is a constant $B$ such that

$$
B f^{*} \omega \leq \operatorname{Ric} f^{*} \omega \text {. }
$$

Assume $0 \notin \operatorname{Ram}_{f}$. Let $b_{1}=b_{1}\left(T_{f, \omega}\right)$. Then for $r<R$ we have

$$
B T_{f, \omega}(r)+N_{f, \operatorname{Ram}}(r) \leq \frac{1}{2} S\left(T_{f, \omega}, b_{1}, \psi, r\right)-\frac{1}{2} \log \gamma_{f}(0)
$$

for $r \geq r_{1}\left(T_{f, \omega}\right)$ outside a set of measure $\leq 2 b_{0}(\psi)$.

Note that the theorem is formulated for a noncompact manifold, and that the map $f$ is defined on a disc. Under the hypothesis of the theorem, there is no nonconstant holomorphic map of $\mathrm{C}$ into $Y$. The theorem gives an implicit bound for the radius of a disc on which a holomorphic map is defined.

\section{REFERENCES}

[Ad1] W. Adams, Asymptotic diophantine approximations to $e$, Proc. Nat. Acad. Sci. U.S.A. 55 (1966), 28-31.

[AD2] W. ADAMS, Asymptotic diophantine approximations and Hurwitz numbers, Amer. J. Math. 89 (1967), 1083-1108.

[A-L] W. AdAms and S. LANG, Some computations in diophantine approximations, J. Reine Angew. Math. 220 (1965), 163-173.

[Ah] L. AHLFors, The theory of meromorphic curves, Acta. Soc. Sci. Fenn. Nova Ser. A 3(1939/47) no. 4 (1941), 1-31. 
[Br] A. D. Bryuno, Continued fraction expansion of algebraic numbers, $\mathrm{Zh}$. Vichisl Mat. i Mat. Fiz. 4 nr. 2 (1964), 211-221, translated USSR Comput. Math. Phys. 4 (1964), 1-15

[C-G] J. CARLSON and P. GRIFFITHS, A defect relation for equidimensional holomorphic mappings between algebraic varieties, Ann. of Math. (2) 95 (1972), 557-584.

[Ch] S. S. CHERn, Complex analytic mappings of Riemann surfaces. I, AMER. J. MATH. 82 (1960), 323-337.

[Gr] P. GRIFFITHS, Entire holomorphic mappings in one and several complex variables, Ann. Math. Studies vol. 85, Princeton Univ. Press, Princeton, N. J., 1976

[G-K] P. GRIFFITHS AND J. KING, Nevanlinna theory and holomorphic mappings between algebraic varieties, АстА МАтн. 130 (1973), 145-220.

[La1] S. LANG, Report on diophantine approximations, Bull. Soc. Math. FRANCE 93 (1965), 177-192.

[La2] S. Lang, Asymptotic diophantine approximations, Proc. Nat. Acad. ScI. U.S.A. 55 (1966), 31-34.

[La3] S. LANG, Introduction to diophantine approximations, AdDISON-WESLEY, 1966.

[La4] S. LANG, Transcendental numbers and diophantine approximations, BuLL. AMER. MATH. Soc. 77 (1971), 635-677.

[La5] S. LANG, The error term in Nevanlinna theory, Duke Math. J. 56 (1988), 193-218.

[L-T1] S. LANG AND H. TRotTer, Continued fractions of some algebraic numbers, J. Reine ANGeW. Math. 255 (1972), 112-134.

[L-T2] S. Lang and H. Trotter, Addendum to the above, J. Reine Angew MATH. 267 (1974), 219-220.

[Ne] R. Nevanlinna, Analytic functions, Springer-Verlag, 1970 (Revised TRANSLATION OF THE GERMAN EDITION, 1953).

[vN-T] J. von Neumann and B. Tuckerman, Continued fractions expansion of $2^{1 / 3}$, Math. TABles Aids Comput. 9 (1955), 23-24.

[Os1] C. F. OsGood, A number theoretic differential equations approach to generalizing Nevanlinna theory, Indian J. MATH. 23 (1981), 1-15.

[Os2] C. F. OsGood, Sometimes effective Thue-Siegel Roth Schmidt-Nevanlinna bounds, or better, J. NUMBER THEORY 21 (1984), 347-389.

[RDM] R. Richtmyer, M. Devaney and N. Metropolis, Continued fraction expansions of algebraic numbers, NUMER. MATH. 4 (1962), 68-84.

[Ro] K. F. Roth, Rational approximations to algebraic numbers, MATEMATIKA 2 (1955), 1-20.

[Sc] W. Schmidt, Diophantine approximation, Lecture Notes in Math., vol. 785, SPringer-Verlag, Berlin ANd New York, 1980.

[Vo1] P. Vojta, Diophantine approximations and value distribution theory, LECTURE Notes IN MATh., Vol. 1239, Springer-Verlag, BerLin AND NeW YORK, 1987. 
[Vo2] P. Vojta, A refinement of Schmidt's subspace theorem AM. J. Math 111 (1989), PP. 489-518.

[Wo] P. M. WoNG, On the second main theorem of Nevanlinna theory (TO APPEAR).

Department of Mathematics, Yale University, New Haven, CONNECTICUT 06520 
